\title{
VOLUMES, MIDDLE-DIMENSIONAL SYSTOLES, AND WHITEHEAD PRODUCTS
}

\author{
Ivan K. Babenko, Mikhail G. Katz, and Alexander I. Suciu \\ Abstract. Let $X$ be a closed, orientable, smooth manifold of dimension $2 m \geq 6$ \\ with torsion-free middle-dimensional homology. We construct metrics on $X$ of \\ arbitrarily small volume, such that every orientable, middle-dimensional subman- \\ ifold of less than unit volume necessarily bounds. Thus, Loewner's theorem has \\ no higher-dimensional analogue.
}

\section{Introduction}

Let $(X, g)$ be a closed, orientable Riemannian manifold of even dimension $2 \mathrm{~m}$. The following notion was introduced by Marcel Berger in [4], [5].

Definition 1.1. The $k$-systole of $(X, g), \operatorname{sys}_{k}(g)$, is the infimum of areas of nonbounding cycles represented by maps of $k$-dimensional manifolds into $X$.

In this note, we will be interested in the following question: Does there exist a constant, $C$, such that every metric $g$ on $X$ satisfies

$$
\operatorname{sys}_{m}^{2}(g) \leq C \cdot \operatorname{vol}_{2 m}(g) ?
$$

If there is no such $C$, we say that $X$ is systolically free.

In the case of surfaces of positive genus the answer to question (1.1) is affirmative. For a history of the problem (dating to C. Loewner's solution in the case $X=S^{1} \times S^{1}$ ), see M. Berger [5] and P. Sarnak [16]. In the case $m \geq 2$, this question has been referred to by M. Gromov as the "basic systolic problem" ([9], p. 357); see also the subsection "Systolic reminiscences" of [10], section 4.48. Progress on the problem became possible once Gromov described a special family of metrics on $S^{1} \times S^{3}$, and surgical procedures suitable for generalizations ([10], section 4.45); see also [12] and [2].

The purpose of this note is to prove the following result (see Theorem 2.3 for a statement in the more general context of CW-complexes).

Theorem 1.2. Let $X$ be a closed, orientable, smooth manifold of dimension $2 m$. If $m \geq 3$ and $H_{m}(X)$ is torsion-free, then $X$ is systolically free.

An underlying theme of this paper is the influence of homotopy theory on the geometric inequality (1.1). The basic idea is to establish the systolic freedom of a

Received July 21, 1997.

1991 Mathematics Subject Classification. Primary 53C23; Secondary 55Q15.

Key words and phrases. volume, systole, systolic freedom, coarea inequality, isoperimetric inequality, Whitehead product, Hilton-Milnor theorem.

The third author was partially supported by N.S.F. grant DMS-9504833. 
complicated manifold, $X$, by "folding away" some of the topology of $X$ into lowerdimensional cells attached to a simpler manifold, $Y$, whose systolic freedom is already established. The notion of "folding away" is captured in Definition 2.4 of a "meromorphic map," $f: X--\rightarrow Y$. The starting point is Gromov's sequence of metrics (2.3), which establishes the systolic freedom of products of spheres.

Our topological tools are, on one hand, the Hilton-Milnor theorem calculating homotopy groups of a wedge of spheres, and, on the other hand, theorems of B. Eckmann and G. Whitehead on composition maps in homotopy groups of spheres. Our geometric tools are the coarea inequality of [8] and pullback arguments for simplicial metrics as described by the first author in [1].

The case $m=2$ of (1.1) remains open, but it has been reduced to either $\mathbb{C P}^{2}$ or $S^{2} \times S^{2}$ by the first two authors in [2]. Even if we restrict the class of competing metrics to homogeneous ones, the inequality (1.1) is violated in certain cases such as $S^{3} \times S^{3}$, see [13].

One may view our construction as a way of producing metrics for which the systole and the mass in middle dimension do not agree, compare [7].

An announcement of this paper appeared in [13].

The structure of the paper is as follows:

- In section 2, we define "meromorphic maps" between regular CW-complexes, which allows us to correlate their systolic freedom. We use this technique to give a short proof of the systolic freedom of $S^{m} \times S^{m}$.

- In section 3, we find maps from the $(2 m-1)$-skeleton of $X$ to a wedge of $m$-spheres that induce monomorphisms in $H_{m}(-, \mathbb{Q})$, and self-maps of $\vee S^{m}$ that send $\pi_{2 m-1}\left(\vee S^{m}\right)$ to the subgroup generated by Whitehead products.

- In section 4 , we prove our theorem in the case where $b_{m}(X)=1$, by mapping $X$ meromorphically to $S^{m} \times S^{m}$.

- In section 5, we present the proof in the general case. This is achieved by mapping $X$ meromorphically to the $2 m$-skeleton of a product of sufficiently many $m$-spheres.

\section{Systolic freedom of CW-complexes and meromorphic maps}

In order to prove Theorem 1.2, we will enlarge the class of manifolds to that of piecewise smooth, simplicial complexes, for which one can still define metrics, volumes, and systoles. We will actually prove our theorem in the context of finite, regular CW-complexes. Such a complex $K$ can be triangulated so that the resulting simplicial complex is a subdivision of $K$ (see [14], p. 80).

Definition 2.1. A finite, regular CW-complex $K$ of dimension $2 m$ is called systolically free if

$$
\inf _{g} \frac{\operatorname{vol}_{2 m}(g)}{\operatorname{sys}_{m}^{2}(g)}=0
$$

where the infimum is taken over all metrics $g$ on $K$. This amounts to the existence of a sequence of metrics $\left\{g_{j}\right\}$ such that

$$
\operatorname{sys}_{m}^{2}\left(g_{j}\right) \geq j \operatorname{vol}_{2 m}\left(g_{j}\right) .
$$


Remark 2.2. The systolic freedom of $K$ (or the absence thereof) is independent of the piecewise smooth simplicial structure that one chooses in its homotopy type. This independence is verified by means of the simplicial approximation theorem and by the pullback arguments for metrics from [1], [2].

Theorem 2.3. Let $K$ be a finite, regular $C W$-complex of dimension $2 m \geq 6$. If $H_{m}(K)$ is torsion-free, then $K$ is systolically free.

This theorem (which slightly generalizes Theorem 1.2), will be proved at the end of section 5. The key to the proof is the following notion, inspired by complex analysis and surgery theory.

Definition 2.4. Let $X$ and $Y$ be $2 m$-dimensional CW-complexes. A "meromorphic map" from $X$ to $Y$ is a continuous map $f: X \rightarrow W$ such that

(i) $W$ is a CW-complex obtained from $Y$ by attaching cells of dimension at most $2 m-1$;

(ii) $f_{*}: H_{m}(X) \rightarrow H_{m}(W)$ is a monomorphism.

We shall denote such "maps" by $f: X--\rightarrow Y$, and drop the quotation marks.

Example 2.5. Let $X$ be a complex surface and $\widehat{X} \rightarrow X$ its blow-up at a point $p \in X$. Then the classical meromorphic map $X \rightarrow \widehat{X}$ can be modified in a neighborhood of $p$ and extended to a continuous map from $X$ to $\widehat{X} \cup_{f} B^{3}$ where the 3 -ball is attached along the exceptional curve.

Proposition 2.6 ([2]). Let $X$ and $Y$ be finite, regular $C W$-complexes. Suppose $X$ admits a meromorphic map to $Y$. If $Y$ is systolically free, then $X$ is also systolically free.

Proof (sketch). Let $f: X \rightarrow W=Y \cup \bigcup_{i} B_{i}^{k_{i}}$ be the given meromorphic map. The attached cells (of dimension $k_{i}<2 m$ ) do not affect the $2 m$-dimensional volume, and thus $W$ is still systolically free, by the cylinder construction of [2], Lemma 6.1. We now pull back to $X$ the systolically free metrics on $W$. Thus $X$ is systolically free.

Proposition 2.7. Let $X$ be an orientable, smooth manifold of dimension $n=$ $2 m$. Suppose $Y$ is obtained from $X$ by performing surgery on embedded, framed $k$-spheres, with $1 \leq k<m$. Then there exists a meromorphic map from $X$ to $Y$.

Proof. Let $W^{n+1}$ be the cobordism between $X$ and $Y$ defined by the surgeries. We claim that the inclusion $X \hookrightarrow W$ is the desired meromorphic map. Indeed, $W$ is obtained by attaching handles $D^{k+1} \times D^{n-k}$ to $X \times I$, or dually, by attaching handles $D^{n-k} \times D^{k+1}$ to $Y \times I$. Hence, $W$ has the homotopy type of $Y$, with cells of dimension $n-k \leq n-1$ attached to it, and so condition (i) is satisfied. Since $k<m$, condition (ii) is satisfied also.

The main geometric ingredient in the proof of Theorem 2.3 is the special case of a product of spheres, first proved in [12]. We provide a different proof in Proposition 2.8 below, using $\mathrm{CW}$-complexes and meromorphic maps. 
In order to give the reader a heuristic understanding of the fundamental examples, let us begin by describing Gromov's construction of systolically free metrics on $S^{1} \times S^{3}$, see [10], section 4.45. We translate Gromov's succinct example, from the language of global Riemannian geometry into the dual language of differential forms.

Let $S^{1}$ be the unit circle with standard 1-form $d z$, and let $S^{3}$ be the unit 3 -sphere with standard contact 1-form $b$. Gromov's metrics $\left\{g_{j}\right\}$ are obtained by modifying the product metric of a circle of length $2 \pi$ with a 3 -sphere of radius $R=\sqrt{1+j^{2}}$, by adding a non-diagonal term $-2 j b d z$ (symmetric tensor product). Explicitly, let us complete $b$ to a basis $\left(b, b^{\prime}, b^{\prime \prime}\right)$ of 1 -forms which is orthonormal with respect to the metric of unit radius, so that $d b=b^{\prime} \wedge b^{\prime \prime}$. Then Gromov's metrics are given by:

$$
g_{j}=(d z-j b)^{2}+b^{2}+R^{2}\left(b^{\prime 2}+b^{\prime \prime 2}\right) .
$$

Roughly speaking, from the point of view of an individual Hopf fiber of $S^{3}$, each metric looks like a square torus of size $2 \pi$ with respect to a judiciously chosen basis. Meanwhile, from the point of view of the hypersurface $S^{3}$ of radius $R$, the metric $g_{j}$ looks like a family of metrics on $S^{3}$ parametrized by a circle of length $\frac{2 \pi}{R}$.

Let $*$ be the Hodge star operator of the metric $g_{j}$. Then:

$$
\begin{aligned}
*(d z)=*(d z-j b)+j * b & =(b-j(d z-j b)) \wedge R^{2} d b \\
& =R^{4} b \wedge d b-j R^{2} d z \wedge d b .
\end{aligned}
$$

The form $* d z$ is closed, since $d * d z=R^{4} d b \wedge d b=0$. We normalize $* d z$ to obtain a calibrating form $\frac{1}{R} * d z$, whose restriction to the 3 -sphere $S^{3} \subset S^{1} \times S^{3}$ coincides with the volume form $R^{3} b \wedge d b$ of this round sphere of radius $R$. Estimating the volume and the systoles yields:

$$
\lim _{j \rightarrow \infty} \frac{\operatorname{vol}_{4}\left(g_{j}\right)}{\operatorname{sys}_{1}\left(g_{j}\right) \cdot \operatorname{sys}_{3}\left(g_{j}\right)}=\lim _{j \rightarrow \infty} \frac{j^{2}}{1 \cdot j^{3}}=0 .
$$

This example generalizes to all products of spheres, except $S^{1} \times S^{1}$ and possibly $S^{2} \times S^{2}$, see [12], [3], [15], [2]. For each such product $S^{m} \times S^{k}$, there is a sequence of metrics $\left\{g_{j}\right\}$ such that

$$
\lim _{j \rightarrow \infty} \frac{\operatorname{vol}_{m+k}\left(g_{j}\right)}{\operatorname{sys}_{k}\left(g_{j}\right) \operatorname{sys}_{m}\left(g_{j}\right)}=0
$$

The existence of metrics on $S^{m} \times S^{k}$ satisfying (2.6) is proved in two steps, first for $m>k$, and next for $m=k$. The first step is done by direct geometric construction, see [2], proof of Proposition 4.2. The second step was actually done beforehand, in [12]. In order to give a flavor of the arguments involved, we provide a self-contained proof of the second step, assuming the first step.

Proposition 2.8 ([12]). For $m \geq 3$, the product $S^{m} \times S^{m}$ is systolically free. 
Proof. Choose an integer $k$ such that $1 \leq k<m$. Performing surgery on a standard $S^{k} \subset S^{m}$ yields $S^{k} \times S^{m-k}$. Let $f: S^{m}--\rightarrow S^{k} \times S^{m-k}$ be the associated meromorphic map, and take the map

$$
\operatorname{id} \times f: S^{m} \times S^{m}--\rightarrow S^{m} \times S^{k} \times S^{m-k} .
$$

By Proposition 2.6, it suffices to prove that the manifold $X=S^{m} \times S^{k} \times S^{m-k}$ is systolically free. Let $\left\{g_{j}\right\}$ be a sequence of metrics on $S^{m} \times S^{k}$ as in (2.6). Let $h_{j}$ be a metric on $S^{m-k}$ of volume $\operatorname{vol}_{m-k}\left(h_{j}\right)=\frac{\operatorname{sys}_{m}\left(g_{j}\right)}{\operatorname{sys}_{k}\left(g_{j}\right)}$. Consider the metric $g_{j} \oplus h_{j}$ on $X$, and let $z$ be a cycle representing a non-zero multiple of $\left[S^{k} \times S^{m-k}\right]$. Let $p: X \rightarrow S^{m-k}$ be the projection to the last factor. By the coarea inequality, we obtain the following lower bound for the volume of $z$ in $\left(X, g_{j} \oplus h_{j}\right)$ :

$$
\begin{aligned}
\operatorname{vol}_{m}(z) & \geq \int_{\left(S^{m-k}, h_{j}\right)} \operatorname{vol}_{k}\left(z \cap p^{-1}(x)\right) d x \geq \operatorname{vol}_{m-k}\left(h_{j}\right) \operatorname{sys}_{k}\left(g_{j}\right) \\
& =\operatorname{sys}_{m}\left(g_{j}\right)
\end{aligned}
$$

where the middle inequality uses intersection numbers for cycles and transversality arguments in the context of maps of manifolds into $X$ ( $c f$. Definition 1.1 and [2], Lemma 6.1.). Hence

$$
\frac{\operatorname{vol}_{2 m}\left(g_{j} \oplus h_{j}\right)}{\operatorname{sys}_{m}^{2}\left(g_{j} \oplus h_{j}\right)}=\frac{\operatorname{vol}_{m-k}\left(h_{j}\right) \operatorname{vol}_{m+k}\left(g_{j}\right)}{\operatorname{sys}_{m}^{2}\left(g_{j}\right)}=\frac{\operatorname{vol}_{m+k}\left(g_{j}\right)}{\operatorname{sys}_{k}\left(g_{j}\right) \operatorname{sys}_{m}\left(g_{j}\right)} \underset{j \rightarrow \infty}{\longrightarrow} 0,
$$

proving the systolic freedom of $X$ and therefore that of $S^{m} \times S^{m}$.

\section{Whitehead products and maps to wedges of spheres}

In this section, we establish some lemmas that will be needed in the proof of Theorem 2.3. The main idea is to use high-degree self-maps of the $m$-sphere $S^{m}$ as in R. Thom [18] to handle torsion in homotopy. In what follows, we will denote a wedge of $b$ copies of $S^{m}$ by $\vee^{b} S_{r}^{m}$, or simply $\vee S^{m}$.

A basic tool is the Hilton-Milnor theorem, which computes the homotopy groups of a wedge of spheres in terms of the homotopy groups of the factors. In particular, it gives the following splittings (see e.g. [20]):

$$
\begin{aligned}
\pi_{k}\left(\vee S_{r}^{m}\right)=\bigoplus_{r} \pi_{k}\left(S_{r}^{m}\right) & \text { for } k \leq 2 m-2, \\
\pi_{k}\left(\vee S_{r}^{m}\right)=\bigoplus_{r} \pi_{k}\left(S_{r}^{m}\right) \oplus \bigoplus_{r<s} \mathbb{Z}\left[e_{r}, e_{s}\right], & \text { for } k=2 m-1,
\end{aligned}
$$

where $\left[e_{r}, e_{s}\right]$ is the Whitehead product of the fundamental classes of the corresponding spheres.

Another tool that we need is a formula of B. Eckmann [6] and G. Whitehead [19] on the "distributive law" in maps from spheres to spheres. Let $\phi_{q}$ : 
$S^{m} \rightarrow S^{m}$ be a map of degree $q$. Let $\phi_{q_{\sharp}}: \pi_{k}\left(S^{m}\right) \rightarrow \pi_{k}\left(S^{m}\right)$ be the induced map. Then, if $x \in \pi_{k}\left(S^{m}\right)$,

$$
\begin{aligned}
\phi_{q_{\sharp}}(x)=q x & \text { for } k \leq 2 m-2, \\
\phi_{q_{\sharp}}(x)=q x+\left(\begin{array}{l}
q \\
2
\end{array}\right) H(x)[e, e] & \text { for } k=2 m-1,
\end{aligned}
$$

where $H(x) \in \mathbb{Z}$ is the Hopf invariant of $x$, and $e \in \pi_{m}\left(S^{m}\right)$ is the fundamental class, see [20], p. 537.

Let $K$ be a finite CW-complex of dimension $2 m-1$. Let $K^{i}$ be the $i$-skeleton of $K$.

Lemma 3.1. There exists a map $f: K \rightarrow \vee^{b} S^{m}$, where $b=\operatorname{rank} H_{m}(K /$ $K^{m-1}$ ), such that

$$
f_{*}: H_{m}(K) / \operatorname{tors}\left(H_{m}(K)\right) \rightarrow H_{m}\left(\bigvee^{b} S^{m}\right) \text { is injective. }
$$

Proof. From the exact sequence of the pair $\left(K, K^{m-1}\right)$ we see that the homomorphism $H_{m}(K) \rightarrow H_{m}\left(K / K^{m-1}\right)$ is injective. This reduces the problem to the case when $K$ is $(m-1)$-connected. If $m=1$, there is a homotopy equivalence $f: K \rightarrow \vee^{b} S^{1}$, and we are done. Thus, we may also assume that $m \geq 2$.

Let $H_{m}(K) \rightarrow \mathbb{Z}^{b}, b=b_{m}(K)$, be the quotient by the torsion subgroup. Since $K$ is $(m-1)$-connected, this homomorphism is realized by a map to the corresponding Eilenberg-MacLane space, $f: K \rightarrow K\left(\mathbb{Z}^{b}, m\right)$. Now $K\left(\mathbb{Z}^{b}, m\right)=$ $(K(\mathbb{Z}, m))^{b}$, and $K(\mathbb{Z}, m)$ is obtained from $S^{m}$ by adding cells in dimensions $m+2$ and higher. Hence, by the cellular approximation theorem, we get a map $f_{m+1}: K^{m+1} \rightarrow \vee^{b} S^{m}$ by restricting to $(m+1)$-skeleta. Clearly, this map satisfies (3.5). Thus, if $m=2$, we are done.

If $m \geq 3$, we extend the map $f_{m+1}$ to $f=f_{2 m}: K^{2 m} \rightarrow \vee^{b} S^{m}$ by induction on skeleta, proceeding as in Serre [17], pp. 278 and 287-288. Given $i$ with $m+1 \leq i \leq$ $2 m-2$, let $f_{i}: K^{i} \rightarrow \vee^{b} S^{m}$ be a map satisfying (3.5). By [17], the group $\pi_{i}\left(S^{m}\right)$ is finite; let $q$ be its order. Then, by (3.1) and (3.3), the map $\left(\vee \phi_{q}\right) \circ f_{i}$ extends to a map $f_{i+1}: K^{i+1} \rightarrow \vee^{b} S^{m}$. Clearly, $f_{i+1}$ satisfies (3.5). This completes the inductive step, and the proof.

Lemma 3.2. A wedge of $m$-spheres, $m \geq 2$, admits a self-map $\phi: \vee^{b} S^{m} \rightarrow$ $\vee^{b} S^{m}$ such that

(i) The map $\phi_{\sharp}: \pi_{2 m-1}\left(\vee^{b} S^{m}\right) \rightarrow \pi_{2 m-1}\left(\vee^{b} S^{m}\right)$ has image contained in the subgroup generated by all the Whitehead products;

(ii) The map $\phi_{*}: H_{m}\left(\bigvee^{b} S^{m}\right) \rightarrow H_{m}\left(\bigvee^{b} S^{m}\right)$ is injective.

Proof. For $b=1$, every Whitehead product is proportional to $[e, e]$, where $e$ is the fundamental class of $S^{m}$. Let $x \in \pi_{2 m-1}\left(S^{m}\right)$. Then we can write

$$
2 x=s+H(x)[e, e]
$$


for some $s$ of finite order. Consider the map $\phi=\phi_{q}: S^{m} \rightarrow S^{m}$, with $q$ even. Substituting (3.6) into (3.4), we obtain:

$$
\begin{aligned}
\phi_{\sharp}(x) & =\frac{q}{2}(s+H(x)[e, e])+\left(\begin{array}{l}
q \\
2
\end{array}\right) H(x)[e, e] \\
& =\frac{q}{2} s+\frac{q^{2}}{2} H(x)[e, e] .
\end{aligned}
$$

Thus, it suffices to take

$$
q=2\left|\operatorname{tors}\left(\pi_{2 m-1}\left(S^{m}\right)\right)\right| .
$$

Alternatively, a theorem of G. Whitehead [19] insures that the subgroup of $\pi_{2 m-1}\left(S^{m}\right)$ generated by Whitehead products is precisely the kernel of the suspension homomorphism $E: \pi_{2 m-1}\left(S^{m}\right) \rightarrow \pi_{2 m}\left(S^{m+1}\right)$. Thus, it suffices to pick $q=\left|\pi_{2 m}\left(S^{m+1}\right)\right|$, which is less than or equal to the value from (3.8).

For $b>1$, we pick $\phi=\vee^{b} \phi_{q}$, with $q$ as in (3.8). The splitting from (3.2) and an argument as above insure that $\phi$ satisfies (i) and (ii).

Remark 3.3. For $m$ odd, $m \geq 3$, we can actually choose $q$ so that $\phi_{\sharp}=0$, since, in that case, $\pi_{2 m-1}\left(S^{m}\right)$ is a finite group, and all its elements have Hopf invariant 0 .

Remark 3.4. Let $\mathbb{F}$ be $\mathbb{C}, \mathbb{H}$ or $\mathbf{C a}$, and let $K=\mathbb{F P}^{2}$ be the corresponding projective plane. With the usual decomposition into 3 cells for $K$, we have $K^{2 m-1}=\mathbb{F P}^{1}=S^{m}$, where $m=\operatorname{dim}(\mathbb{F})$. The smallest positive integer $q$ for which $\phi_{q}: S^{m} \rightarrow S^{m}$ satisfies conditions (i) and (ii) from Lemma 3.2 can be computed explicitly in these examples. Recall that $\pi_{2 m-1}\left(S^{m}\right)=\mathbb{Z} \oplus T_{m}$, where $T_{m}$ is a finite cyclic group, of order equal to 1,12 , or 120 respectively when $\mathbb{F}$ is $\mathbb{C}, \mathbb{H}$, or $\mathbf{C a}$. Let $a$ be the infinite order generator defined by the Hopf map, and let $s$ be a generator of the torsion part (taken to be 0 when $\mathbb{F}=\mathbb{C}$ ). A result of H. Toda (see [11]) states that $[e, e]=2 a \mp s$. From this formula and (3.7) we obtain for $q$ even:

$$
\phi_{q \sharp}(a)=\frac{q}{2} 2 a+\left(\begin{array}{l}
q \\
2
\end{array}\right)[e, e]= \pm \frac{q}{2} s+\frac{q^{2}}{2}[e, e] .
$$

Thus, the necessary and sufficient condition for (i) and (ii) to hold is that $q$ be a non-zero multiple of $2\left|T_{m}\right|$.

\section{Meromorphic maps to $S^{m} \times S^{m}$}

In this section, we prove Theorem 2.3 in the particular case where $H_{m}\left(K / K^{m-1}\right)=\mathbb{Z}$, by constructing a meromorphic map $h: K--\rightarrow S^{m} \times S^{m}$. The essential ingredients of the general case are already present here, but the proof is more transparent in this simpler situation.

Proof of Theorem 2.3 (particular case). Let $K$ be a finite, regular CW-complex of dimension $2 m \geq 6$. Assume $H_{m}\left(K / K^{m-1}\right)=\mathbb{Z}$.

By Lemma 3.1, there is a map $f: K^{2 m-1} \rightarrow S^{m}$ such that $f_{*}: H_{m}\left(K^{2 m-1} /\right.$ $\left.K^{m-1}\right) \rightarrow H_{m}\left(S^{m}\right)$ is injective. By Lemma 3.2, there is a map $\phi=\phi_{q}^{m}$ : 
$S^{m} \rightarrow S^{m}$ of degree $q \neq 0$ that maps $\pi_{2 m-1}\left(S^{m}\right)$ to the subgroup generated by the Whitehead product $[e, e]$. The map $\phi \circ f: K^{2 m-1} \rightarrow S^{m}$ also maps $\pi_{2 m-1}\left(K^{2 m-1}\right)$ to this subgroup, while inducing a monomorphism on $H_{m}$.

Now let $a_{1}$ and $a_{2}$ be the generators of $\pi_{m}\left(S^{m} \times S^{m}\right)$, corresponding to the inclusions of the factors. Recall that $S^{m} \times S^{m}=S^{m} \vee S^{m} \cup_{\left[a_{1}, a_{2}\right]} B^{2 m}$. Attaching an $(m+1)$-cell along the diagonal map $(1,1): S^{m} \rightarrow S^{m} \times S^{m}$, we obtain the $2 m$-dimensional regular CW-complex

$$
W=S^{m} \times S^{m} \cup_{a_{1}+a_{2}} B^{m+1} .
$$

Since $m \geq 2$, the complex $W$ satisfies condition (i) in Definition 2.4. Let $\alpha$ : $S^{m} \rightarrow W$ be the composite $S^{m} \stackrel{(1,0)}{\rightarrow} S^{m} \times S^{m} \hookrightarrow W$. Then

$$
\begin{aligned}
\alpha([e, e]) & =\left[a_{1}, a_{1}\right] & & \text { since } \alpha(e)=a_{1} \\
& =\left[a_{1},-a_{2}\right] & & \text { since } a_{1}+a_{2}=0 \text { in } \pi_{m}(W) \\
& =0 & & \text { since }\left[a_{1}, a_{2}\right]=0 \text { in } \pi_{2 m-1}\left(S^{m} \times S^{m}\right) .
\end{aligned}
$$

Now let $h: K^{2 m-1} \rightarrow W$ be the composite $K^{2 m-1} \stackrel{f}{\rightarrow} S^{m} \stackrel{\phi}{\rightarrow} S^{m} \stackrel{\alpha}{\rightarrow} W$. By the above, $h_{\sharp}: \pi_{2 m-1}\left(K^{2 m-1}\right) \rightarrow \pi_{2 m-1}(W)$ is the 0 map. Thus, $h$ extends over the $2 m$-cells of $K$, to a map $h: K \rightarrow W$. Since $h$ is clearly injective on $H_{m}$, we have defined a meromorphic map from $K$ to $S^{m} \times S^{m}$. By Proposition 2.8, the manifold $S^{m} \times S^{m}$ is systolically free. Hence, by Proposition 2.6, the complex $K$ is also systolically free.

\section{Meromorphic maps to skeleta of products of spheres}

Before proving the general case of Theorem 2.3, we establish the systolic freedom of a model space by a "long cylinder" argument.

Let $X$ be a triangulated manifold of dimension $n$. Let $A$ be the $n$-skeleton of $X \times I$ where $I$ is an interval. Then $A=X \times \partial I \cup X^{n-1} \times I$. Let $g_{+}$and $g_{-}$ be two metrics on $X$, and $g_{0}$ another metric dominating both $g_{+}$and $g_{-}$. Let $g$ be the metric on $A$ obtained by restricting the metric $g_{t} \oplus d t^{2}$ of $X \times I$, where $I=[-L, L]$, with $L=\ell+1>1$, and

$$
g_{t}= \begin{cases}g_{0} & \text { if }|t| \leq \ell, \\ (1-\lambda) g_{0}+\lambda g_{ \pm} & \text {if } t= \pm(\ell+\lambda), \text { with } 0 \leq \lambda \leq 1 .\end{cases}
$$

Lemma 5.1. For $k \geq 2$ and $\ell$ sufficiently large, we have $\operatorname{sys}_{k}(g) \geq \beta$, where

$$
\beta=\frac{1}{2} \min \left(\operatorname{sys}_{k}\left(g_{+}\right), \operatorname{sys}_{k}\left(g_{-}\right)\right) .
$$

Proof. Suppose $z$ is a non-bounding $k$-cycle in $A$ such that $\operatorname{vol}_{k}(z)<\beta$. Let $p: A \rightarrow[0, L]$ be the restriction of the map $X \times I \rightarrow[0, L]$ given by $(x, t) \mapsto|t|$. The coarea inequality yields a point $t_{0} \in[0, \ell]$ such that the $(k-1)$-cycle $\gamma=$ $z \cap p^{-1}\left(t_{0}\right)$ satisfies

$$
\operatorname{vol}_{k-1}(\gamma) \leq \frac{1}{\ell} \operatorname{vol}_{k}(z)
$$


By the isoperimetric inequality for cycles of small volume ([8], Sublemma 3.4. $\left.\mathrm{B}^{\prime}\right)$ applied to $\left.g_{0}\right|_{X^{n-1}}$, there is a constant $C=C\left(\left.g_{0}\right|_{X^{n-1}}\right)$ with the following property: Every $(k-1)$-cycle $\gamma$ in $X^{n-1}$ with $\operatorname{vol}(\gamma)<\frac{1}{C}$ bounds a $k$-chain $D$ in $X^{n-1}$, of volume

$$
\operatorname{vol}_{k}(D) \leq C \operatorname{vol}_{k-1}(\gamma)^{\frac{k}{k-1}}
$$

By choosing $\ell>\beta C$ we insure that the isoperimetric inequality applies to $\gamma$. Moreover, we need to choose $\ell$ so that $\operatorname{vol}_{k}(D)<\beta$. Thus we also require $C\left(\frac{\beta}{\ell}\right)^{\frac{k}{k-1}}<\beta$, that is, $\ell>\sqrt[k]{\beta C^{k-1}}$.

Write $D=D_{-}+D_{+}$where $D_{ \pm} \subset X^{n-1} \times\left\{ \pm t_{0}\right\}$. Consider the decomposition of $z$ into a sum of cycles, $z=z_{-}+z_{0}+z_{+}$, where $z_{-}=p^{-1}\left(\left[0, t_{0}\right]\right) \cap(X \times[-L, 0])+$ $D_{-}, z_{0}=p^{-1}\left(\left[0, t_{0}\right]\right)+D_{+}-D_{-}$, and $z_{+}=p^{-1}\left(\left[0, t_{0}\right]\right) \cap(X \times[0, L])-D_{+}$. Now let $\epsilon=0,+$, or - . We have

$$
\operatorname{vol}_{k}\left(z_{\epsilon}\right) \leq \operatorname{vol}_{k}(z)+\operatorname{vol}_{k}(D)<\beta+\beta=\min \left(\operatorname{sys}_{k}\left(g_{+}\right), \operatorname{sys}_{k}\left(g_{-}\right)\right) .
$$

Hence $z_{\epsilon}$ is a boundary for every $\epsilon$ and so $[z]=0$. The contradiction proves the lemma.

Lemma 5.2. Let $B=\left(\times^{c} S^{m}\right)^{2 m}$ be the $2 m$-dimensional skeleton of a product of $c$ copies of the $m$-sphere, $c \geq 2, m \geq 3$. Then $B$ is systolically free.

Proof. We choose the following representative $B_{0}$ in the homotopy class of $B$. Take the Cartesian product of the wedge $\vee^{c} S^{m}$ with the wedge of $\left(\begin{array}{l}c \\ 2\end{array}\right)$ intervals $I_{r s}=[0, L]$ for sufficiently large $L=\ell+1$. At the end of each interval, attach a $2 m$-cell along the Whitehead product, $\left[e_{r}, e_{s}\right]$, of the fundamental classes of the spheres $S_{r}^{m} \times\{L\}$ and $S_{s}^{m} \times\{L\}$ in $\vee^{c} S^{m} \times I_{r s}$ :

$$
B_{0}=\left(\bigvee^{c} S^{m}\right) \times\left(\bigvee_{r<s} I_{r s}\right) \cup \bigcup_{r<s} D_{r s}^{2 m}
$$

We precompose the projection $p: \vee I_{r s} \rightarrow[0, L]$ with the projection to the second factor of $\vee S^{m} \times \vee I_{r s}$ and extend it to a map $p: B_{0} \rightarrow[0, L]$ by setting $p\left(D_{r s}^{2 m}\right)=L$.

Now we apply the argument of Lemma 5.1 with $n=2 m$ to $B_{0}$, as follows. Identify each cell closure $X_{r s}=\bar{D}_{r s}^{2 m}$ with $S^{m} \times S^{m}$ by means of a diffeomorphism $X_{r s} \stackrel{\rho_{r s}}{\longrightarrow} S^{m} \times S^{m}$. Then pull back a sequence of free metrics $\left\{g_{j}\right\}$ on $S^{m} \times S^{m}$ provided by Proposition 2.8 to obtain a sequence of free metrics $\left\{\rho_{r s}^{*}\left(g_{j}\right)\right\}$ on $X_{r s}$, which play the role of the metrics $g_{ \pm}$from Lemma 5.1.

Let $\left(X^{n-1}, g_{0}\right)$ be the wedge of round spheres $S^{m} \vee S^{m}$ of sufficiently big radius so that $g_{0}$ dominates all of the metrics $\left.\rho_{r s}^{*}\left(g_{j}\right)\right|_{X^{n-1}}$. We obtain a lower bound for the $m$-volume of a non-bounding $m$-cycle $z$ in $B_{0}$ by means of a decomposition $z=z_{0}+\sum_{r<s} z_{r s}$, where $z_{r s}$ is a cycle in $\vee^{c} S^{m} \times I_{r s} \cup_{\left[e_{r}, e_{s}\right]} D_{r s}^{2 m} \subset B_{0}$. This decomposition is obtained by the coarea inequality applied to the projection $p: B_{0} \rightarrow[0, L]$. This proves the systolic freedom of $B_{0}$, and hence that of $B$, by Remark 2.2. 
Proof of Theorem 2.3 (general case). Let $K$ be a finite, regular CW-complex of dimension $2 m \geq 6$, with tors $\left(H_{m}(K)\right)=0$. Let $f: K^{2 m-1} \rightarrow \vee^{b} S^{m}$ be a map as in Lemma 3.1, and let $\phi: \vee^{b} S_{r}^{m} \rightarrow \vee^{b} S^{m}$ be a map as in Lemma 3.2. Then the composite $\phi \circ f: K^{2 m-1} \rightarrow \vee^{b} S^{m}$ maps $\pi_{2 m-1}\left(K^{2 m-1}\right)$ to the subgroup of $\pi_{2 m-1}\left(\bigvee^{b} S^{m}\right)$ generated by the Whitehead products in the wedge, while inducing a monomorphism on $H_{m}$.

Let $B$ be the $2 m$-skeleton of a product of $c=2 b$ copies of $S^{m}$, as in Lemma 5.2. We construct a meromorphic map from $\vee^{b} S^{m}$ to $B$ much as in the case $b=1$. Namely, let $\alpha_{r}: S_{r}^{m} \rightarrow S_{r}^{m} \times S_{b+r}^{m}$ be the inclusion into the first factor. Let $W$ be the CW-complex obtained by attaching $(m+1)$-cells to $B$ along the "diagonals" $a_{r}+a_{b+r}$. Define $\alpha: \vee^{b} S^{m} \rightarrow W$ to be the composite

$$
\alpha: \vee^{b} S_{r}^{m} \stackrel{\vee^{b} \alpha_{i}}{\longrightarrow} \vee^{b}\left(S_{r}^{m} \times S_{b+r}^{m}\right) \hookrightarrow B \hookrightarrow W,
$$

and let $h=\alpha \circ \phi \circ f: K^{2 m-1} \rightarrow W$. Then $h_{\sharp}$ sends $\pi_{2 m-1}\left(K^{2 m-1}\right)$ to 0 . Therefore, $h$ extends to a map $h: K \rightarrow W$. Clearly, $h_{*}: H_{m}(K) \rightarrow H_{m}(W)$ is injective. Thus, we have defined a meromorphic map from $K$ to $X$. By Lemma 5.2 and Proposition 2.6, $K$ is systolically free.

\section{Acknowledgements}

The seond author is grateful to the Geometry \& Dynamics Program at Tel Aviv University for hospitality during part of the preparation of this work. The third author is grateful to the Institut Élie Cartan at Henri Poincaré UniversityNancy 1 for hospitality during the completion of this work.

\section{References}

1. I. Babenko, Asymptotic invariants of smooth manifolds, Russian Acad. Sci. Izv. Math. 41 (1993), 1-38.

2. I. Babenko and M. Katz, Systolic freedom of orientable manifolds, Ann. Sci. École Norm. Sup. (1998, to appear); available at http://front.math.ucdavis.edu/math.DG/9707102.

3. L. Bérard Bergery and M. Katz, Intersystolic inequalities in dimension 3, Geom. Funct. Anal. 4 (1994), 621-632.

4. M. Berger, Du côté de chez Pu, Ann. Sci. École Norm. Sup. 5 (1972), 1-44.

5. _ Systoles et applications selon Gromov, Séminaire N. Bourbaki, exposé 771, Astérisque 216 (1993), 279-310.

6. B. Eckmann, Ueber die Homotopiegruppen von Gruppenraümen, Comment. Math. Helv. 14 (1941), 234-256.

7. H. Gluck, D. Mackenzie, and F. Morgan, Volume-minimizing cycles in Grassmann manifolds, Duke Math. J. 79 (1995), 335-404.

8. M. Gromov, Filling Riemannian manifolds, J. Differential Geom. 18 (1983), 1-147.

9. _ Systoles and intersystolic inequalities, Actes de la table ronde de géométrie différentielle en l'honneur de Marcel Berger (A. Besse, ed.), Séminaires et Congrès 1, Société Mathématique de France, 1996, pp. 291-362.

10. _ Metric structures for Riemannian and non-Riemannian spaces, Progr. Math., vol. 152, Birkhäuser, Boston, MA, 1998.

11. S.-T. Hu, Homotopy theory, Pure Appl. Math., vol. 8, Academic Press, Boston, MA, 1959.

12. M. Katz, Counterexamples to isosystolic inequalities, Geom. Dedicata 57 (1995), 195-206.

13. __ Systolically free manifolds, Appendix D in [10].

14. A. Lundell and S. Weingram, The topology of CW complexes, University Series in Higher Math., Van Nostrand Reinhold, New York, 1969. 
15. C. Pittet, Systoles on $S^{1} \times S^{n}$, Differential Geom. Appl. 7 (1997), 139-142.

16. P. Sarnak, Extremal geometries, Extremal Riemann surfaces (J. R. Quine and P. Sarnak, eds.), Contemp. Math., vol. 201, Amer. Math. Soc., Providence, RI, 1997, pp. 1-7.

17. J.-P. Serre, Groupes d'homotopie et classes de groupes abeliens, Ann. of Math. 58 (1953), 258-294.

18. R. Thom, Quelques propriétés globales des variétés différentiables, Comment. Math. Helv. 28 (1954), 17-86.

19. G. W. Whitehead, A generalization of the Hopf invariant, Ann. of Math. 51 (1950), 192237.

20. Elements of homotopy theory, Grad. Texts in Math., vol. 61, Springer-Verlag, New York, 1978.

Département des Sciences Mathématiques, Université de Montpellier 2, Place EugÈne Bataillon, 34095 Montpellier, FRANCE

E-mail address: babenko@darboux.math.univ-montp2.fr

UMR 9973, Département de Mathématiques, Université de Nancy 1, B. P. 239, 54506 VANDOEUVRE, FRANCE

E-mail address: katz@iecn.u-nancy.fr

Department of Mathematics, Northeastern University, Boston, MA 02115, USA

E-mail address: alexsuciu@neu.edu 